\title{
ON THE ACTIVATION OF STUDENTS THROUGH AUGMENTED REALITY EXPERIENCES
}

\author{
Yengui, Mohamed Habib; \\ Stechert, Carsten \\ Ostfalia University of Applied Sciences
}

\begin{abstract}
Over the last ten years, several stakeholders from the higher education sector have been involved in the area of student activation. This is due to the fact that, among other things, lecturers can get better feedback from the learners through active learning and thus can make the lecture more goal-oriented. Due to technological progress and currently available learning platforms, interactive methods are often used, which can be carried out with the help of online tools. Among the most commonly used methods are Peer Instruction (PI) and Just in Time Teaching (JiTT).

All kinds of Mixed Reality are increasingly used in industrial and social context, such as product development processes. Through Augmented Reality (AR) technology and the constant availability of powerful mobile devices, 3D-based content can be virtually linked to the real environment. This enables teaching complex content efficiently, which is otherwise difficult to achieve using conventional methods.

This paper shows an example on the creation of an AR-Experience to activate students even in a situation, that prevents attandence learning. The procedure is explained along the design and calculation of a non-switchable clutches within the lecture "machine elements".
\end{abstract}

Keywords: Augmented Reality (AR), Student Activation, Design education, Virtual reality, Design learning

\section{Contact:}

Stechert, Carsten

Ostfalia University of Applied Sciences

Faculty of Mechanical Engineering

Germany

c.stechert@ostfalia.de 


\section{INTRODUCTION}

All kinds of Mixed Reality are increasingly used in industrial and social context, such as product development processes (Balzerkiewitz und Stechert 2020). Through Augmented Reality (AR) technology and the constant availability of powerful mobile devices, 3D-based content can be virtually linked to the real environment. This enables teaching complex content efficiently, which is otherwise difficult to achieve using conventional methods. Since April 2020, the Innovation-Plus project is funded by the Ministry of Science and Culture of the German state of Lower Saxony. Within this framework, various Augmented Reality contents are created at Ostfalia University of Applied Sciences, Faculty of Mechanical Engineering. The main objective is to support university teaching with "efficient learning". To put it more precisely, content that is difficult to understand should be illustrated interactively and usable in a time-independent manner. At the beginning of the Innovation-Plus project, a framework creating AR experiences was prepared: A catalogue of questions and a workflow guides the user through an AR experience creation process. The workflow serves to concretise the objectives of the supervisory board deployment and thus to determine the type of teaching (Chlebusch et al. 2020). On the other hand, the AR experience creation process presents the AR creation software currently available at Ostfalia University of Applied Sciences, its purposes and most important functions. Next, the university will benefit from the developed infrastructure in the mentioned framework by creating targeted AR content to improve the quality of teaching.

Over the last ten years, several stakeholders from the higher education sector have been involved in the area of student activation. This is because, lecturers can get better feedback from the learners through active learning and thus can make the lecture more goal-oriented. Due to technological progress and currently available learning platforms, interactive methods are often used, which can be carried out with the help of online tools. Peer Instruction (PI) and Just in Time Teaching (JiTT) represent two of the most commonly used methods. Since the attendance courses at Ostfalia University of Applied Sciences were cancelled due to the corona pandemic, the above-mentioned activation methods cannot be used optimally at present. However, it is important for online lectures to motivate students through new techniques and to give and receive constructive feedback. For this reason, an interactive method that considers the current situation is being developed for machine elements lecture.

This paper is divided into four chapters. The first chapter describes similar experiences for activating students and thus explains the usual interactive methods and terms used. In the next chapter, a topic from the machine elements lecture is selected to be used as teaching content for the method to be developed. Based on this, the objectives are defined and concretised with the help of a flow chart. First, the basics of the selected topic are described in detail and the necessary calculations are carried out. In the same chapter, the creation of the AR experiences and thus the activating exercise is described. In the last chapter, the use of the developed method is briefly explained and an evaluation survey for the students is suggested. With the help of the assessments, new findings are to be derived and, if necessary, adjustments made.

\section{STATE OF THE ART}

This chapter deals with those methods that have been used in studies or publications to activate students in order to improve university teaching and that are applicable to mechanical engineering studies. The experience and knowledge gained from some studies may prove to be a useful basis for the definition of objectives and the synthesis of the present paper. This is because this and the other papers agree not only on the main objective, namely student activation, but also on similar ideas and approaches (use of activation methods).

As interactive methods, the leaders of these studies have chosen both the classical variants, e.g. Peer Instruction (PI) and Just in Time Teaching (JiTT), and the AR-based alternative. The combination of these methods and their use is becoming day by day easier due to the technological development and thus the IT infrastructure provided. 


\subsection{AR Basics}

Augmented Reality is a technique that enables the three-dimensional combination of virtual content and the real environment, allowing the user to interact with both realities in real time with partial overlap (Azuma 1997). In addition to teaching, augmented reality is well established in several areas, namely in industry, health, entertainment, transport, etc. (Azuma 1997). This is because AR technology makes it possible to show animated 3D models and annotations in a real world and to observe it from all angles. The technology is mobile and cost-effective. In industry, for example, this can save a lot of time and money during the development phase by avoiding the creation of prototypes. AR content can be viewed with various types of hardware, including AR glasses, tablets and even smartphones (Lee 2012).

\subsection{AR Learning}

Augmented Reality can help users to observe multidimensional visualizations in different levels of complexity and to examine the steps resulting from the variable information density in detail. By applying AR, additional information can be added to the 3D model, which contributes to a better understanding of the entire representation. This cannot be achieved by using an image or just a 3D model. In addition, the free movement of the marker in space allows the user to choose the appropriate viewing angle to examine three-dimensional assembly models and compare their individual parts with the real components (Fotis Liarokapis et al. 2004). Figure 1 shows the application of an AR experience, which includes a two-stage gear unit during a "machine elements" lecture.

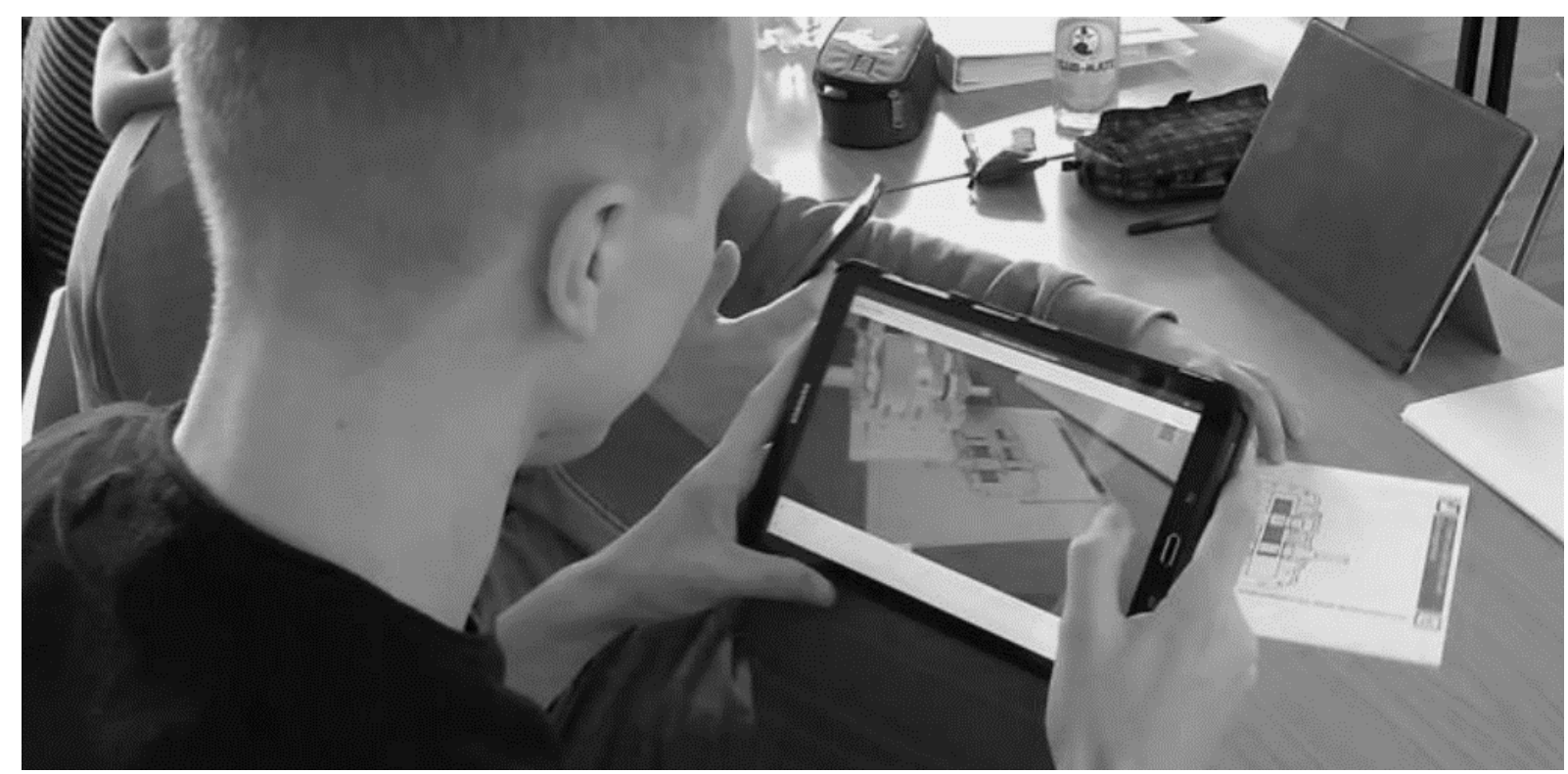

Figure 1: Application of an AR experience which includes a two-stage gear unit during a "machine elements" lecture (Chlebusch et al. 2020)

\subsection{Activation in Higher Education}

Some current challenges of university teaching are the optimal handling of heterogeneous professional requirements of students, building an active learning process and improving feedback between students and teachers. These challenges are complementary and interrelated, as active students can provide better feedback to the teacher and thus help to identify and manage the heterogeneity between students (Wolf et al. 2014). Wolf et al. are convinced that students should also receive feedback on their own performance level and therefore they must actively participate in the course. Feedback on the level of performance can play a particularly important role for first semester students, as they usually do not recognize the difficulties behind the learning material. As a result, they do not begin to work intensively on the subject matter until shortly before the exam phase, which then often fails. This is because intensive self-study leads to bulimic learning. According to Marrs and Novak, the term "bulimic learning" means that the teaching content is treated only superficially due to e.g. time pressure. Thus, learners develop strategies or "recipes to pass" without understanding the deep meaning of the learning material (Marrs und Novak 2004). Nevertheless, mathematical teaching content must be examined more closely and over a longer period of time by the authors so that other 
disciplines can fall back on it and the development of these competencies can be promoted (Novak 1999). Moreover, the studies show that basic learning helps students to retain content longer in their minds, which is highly unlikely in intensive learning. Due to the lack of feedback, lecturers are unable to see whether the students understood the subject matter. Therefore, they cannot find out the level of their students' performance until after the exam. Consequently, the authors believe that these challenges can be met by using the didactic concept that is created by combining the activating methods PI and JITT (Wolf et al. 2014).

\subsection{Peer instruction}

Peer Instruction (PI) was developed by Eric Mazur in the 1990s (Mazur 1997). He used it at that time as part of his university education in physics. This method is performed in two rounds. The first round starts with a multiple-choice question, which is put to the learners about a subject concept. The respondents usually have 5 to 15 minutes to think about the question individually and take part in the voting. Then the students are asked to start a discussion among themselves and to convince the contact person of their own chosen answer. After the discussion phase, the second round and thus a second voting is started, in which the students can revise their first answers based on the discussion. According to Mazur, the correct answer usually prevails through discussion. If not, this would indicate problems of understanding or difficulties with the topic discussed. Therefore, the teacher should provide an explanation. On Eric Mazur's recommendation, the second round of the method does not need to be performed if the rate of correct answers is below $30 \%$ or above $70 \%$. This is probably because the discussion can have a negative effect if the rate is very low, so that even the few students who answered correctly follow the opinion of the majority. On the other hand, if the rate is very high, the effort for the discussion and the second round might not be necessary (Bauer 2019).

\subsection{Just in Time Teaching}

The just in time teaching (JiTT) method is based on the preparation phase. This means that the students use the provided material (e.g. texts, videos, articles, etc.) independently in the preparation of the courses and then solve the accompanying tasks. Of course, learners should be able to solve the uncertainties or note comprehension problems, and then to solve them during the teacher's attendance time to report. With the help of this method, students can learn at their own pace and benefit from needs-oriented assistance. Therefore, the contact time is used more efficiently (Wolf et al. 2014).

\subsection{Summary}

As already mentioned in the introduction of this chapter, all studies or publications considered were concerned with the activation of students to improve the quality of teaching as the main objective. Based on the mentioned studies, this has been achieved relatively successfully, both through the classical methods and through the innovative (AR-based) methods.

Next, the most important success factors as well as the lessons learned from these studies must be considered in the course of the present study work. These can be formulated as follows, including the concrete definition of targeted exercises that lead to cognitive activation of the students. In addition, the exercises should make the students think about the core concepts of the topic dealt with, thus providing a framework for communication and in-depth questions, for example by constructing plausible distractors or integrating effective parameter changes. The psychological factor or motivation should also be aimed for e.g. by presenting exciting and varied AR content during the exercise.

As already mentioned in chapter 1, neither Peer Instruction nor the Just in Time Teaching method is used due to the corona pandemic. However, some characteristics of these methods were adopted in order to develop a similar method, which should be more suitable for online events.

\section{TARGET SETTING}

As explained in the first chapter, the Innovation Plus project was assigned the main objective of efficient learning. Although the application of Augmented Reality is the overall context, each experience developed new sub-goals or new approaches, which obviously depend on the teaching content. As a result, the chapter concretises the main objective of this study work on two levels and describes the subobjectives that result from this. In addition, a flow chart is used to determine the level of difficulty and the type of teaching method for the AR experiences to be produced (Chlebusch et al. 2020). 


\subsection{Hierarchy of objectives and criteria}

This paper helps to take a further step towards realising efficient learning by addressing a topic from the machine elements lecture by activating students, thus also promoting the use of Augmented Reality in university teaching. The calculation and design of clutches was chosen as a topic for AR deployment, because experience and evaluations of recent AR deployments show that students have great difficulties in this area. One reason is that clutches are thought shortly before the examination phase, where students have little time and motivation to practise and understand the conceptual core of the teaching content, see also (Bauer 2019).

The above-mentioned sub-goals are concretised in order to be able to better control their realisation and to avoid misunderstandings. The first sub-goal or learning objective is concretised into the following items.

- The first item is to explain the basics and prerequisites for the design of clutches. The aim here is to provide teaching material for the exercises to be created and to close some gaps in knowledge.

- The second item is to select the type of task, namely the multiple-choice tasks. Distractors (cp. section 4.3) can also be used to encourage students to think about the concept in more depth and, if necessary, to decide on the appropriate formula or approach. Furthermore, this type of task is technically uncomplicated and easy to create.

- Based on the most common errors during the last semesters, it is clear that two additional issues should be considered more intensively when dealing with the topic of clutch design: design against torque shocks and design against periodic alternating torque fluctuations. Therefore, the multiple-choice tasks will exclusively deal with these two issues. This will provide students with both specific assistance and more material to practise.

- The systems and numerical values used in the exercise should correspond to those used in practice. This gives the student a feeling of how important the exercise and the topic in general can be for the future daily work routine, thus increasing motivation and concentration.

The second sub-goal of this study was also set out in six items:

- Firstly, the tools provided by the Innovation Plus project should be used to create an AR experience. This means using the existing flowchart to determine the level of difficulty and type of teaching of the experiences to be created.

- In addition, an AR creation procedure will be followed in order to benefit from the experience already gained in the use of PTC software and from the licences provided by Ostfalia.

- In order to promote the use of AR in university teaching, new creation ideas or widgets should at best be used at each step of the project implementation or for each student research project carried out within the Innovation Plus framework. In this way, new areas of application for AR use can be discovered and students can be offered varied AR content. Furthermore, students are involved in the creation process and bring in their own experiences.

- Due to the corona pandemic, all courses are currently held online. Therefore, individual learning should receive more attention so that students can access teaching and exercise content independent of time and place. AR content is very well suited for this, as it can be stored online and downloaded by students. Furthermore, users can also deal more intensively with the core ideas behind each exercise unit, regardless of the time pressure of the courses.

- According to the experience from the previous steps of the project, the density of information in AR experiences should be kept low. In spite of the extensive exercises in clutch design, this can be achieved with the help of the flowchart or the appropriate teaching method and the fade-in and fade-out functions.

- In order to be further developed and used in teaching, AR should apply in several areas so that its full potential can be realised. In addition, the relevance of AR assignments must be confirmed by the constantly positive evaluations of the students.

\subsection{Application and result of the flow chart for the AR exercise}

First, the degree of complexity of the AR experience must be determined in order to apply the flow chart. This is based on the learning goal taxonomy in the cognitive area according to Bloom (Bloom 1956). This is categorized according to the behavioural components of the learners in six levels of Cognitive Learning Outcomes, as follows: Level 1: Knowledge, Level 2: Understanding, Level 3: 
Application, Level 4: Analysis, Level 5: Synthesis, Level 6: Evaluation/ Assessment (highest level) (Waldherr und Walter 2014), (Rozsa 2012). According to Bloom's categorization, the AR exercise to be created here has reached the highest complexity level, namely "Evaluation / Assessment".

After applying the flow chart for creating AR content, it was decided to develop the experiences from a combination of the four teaching methods, namely 2D, 3D, paper and animation.

\section{CREATION OF ACTIVATING AR-EXERCISE TO DEAL WITH THE TOPIC CLUTCH DESIGN}

In this chapter, the basics for the design of non-switchable clutches are briefly explained first. Subsequently, the task and the concept of the AR exercise are presented. Next, the questions posed are answered and supplemented with distractors, which were formed from frequently observed mistakes made by students. Finally, the most important steps for creating the exercise task and the AR experiences are described, taking into account the AR experience creation process.

\subsection{Basics for the design of non-switchable clutches}

A non-switchable clutch is mainly used to create a rigid connection between an input and an output machine, thus forces and torques can be transmitted. In addition, clutches have various compensation functions, such as reducing shocks and vibrations and compensating for misalignment and positional errors. The compensating functions are considered as additional functions in the design, as they are specific to the clutch and depending on the material, type of connection and geometry.

As shown in Figure 2, the design of non-switchable clutches can be divided into four main steps. First of all, the type is selected with consideration of the system properties. System properties in this context are for example the compliance, operating conditions etc. First, the selected type should be assigned a size using the application factor $K_{A}$.

In the third step, the roughly selected size must be checked against three load types. These are namely torque shocks, periodic alternating torques and shaft displacements.

The check against the first load type must be carried out using the formulas shown in Figure 2. Firstly, the moments of inertia are reduced to the respected clutch. Secondly, the torque is calculated according to the acceleration on the input or output side. For the verification of periodic alternating torques, it must also be known whether the vibration excitation is on the input or output side in order to select the correct formula. To verify the shaft misalignments, the given numerical values only have to be inserted into the formula and compared with the permitted misalignments.

Next, it is checked whether all calculation results are below the permitted numerical values from the clutch manufacturer. If not, another size must be selected.

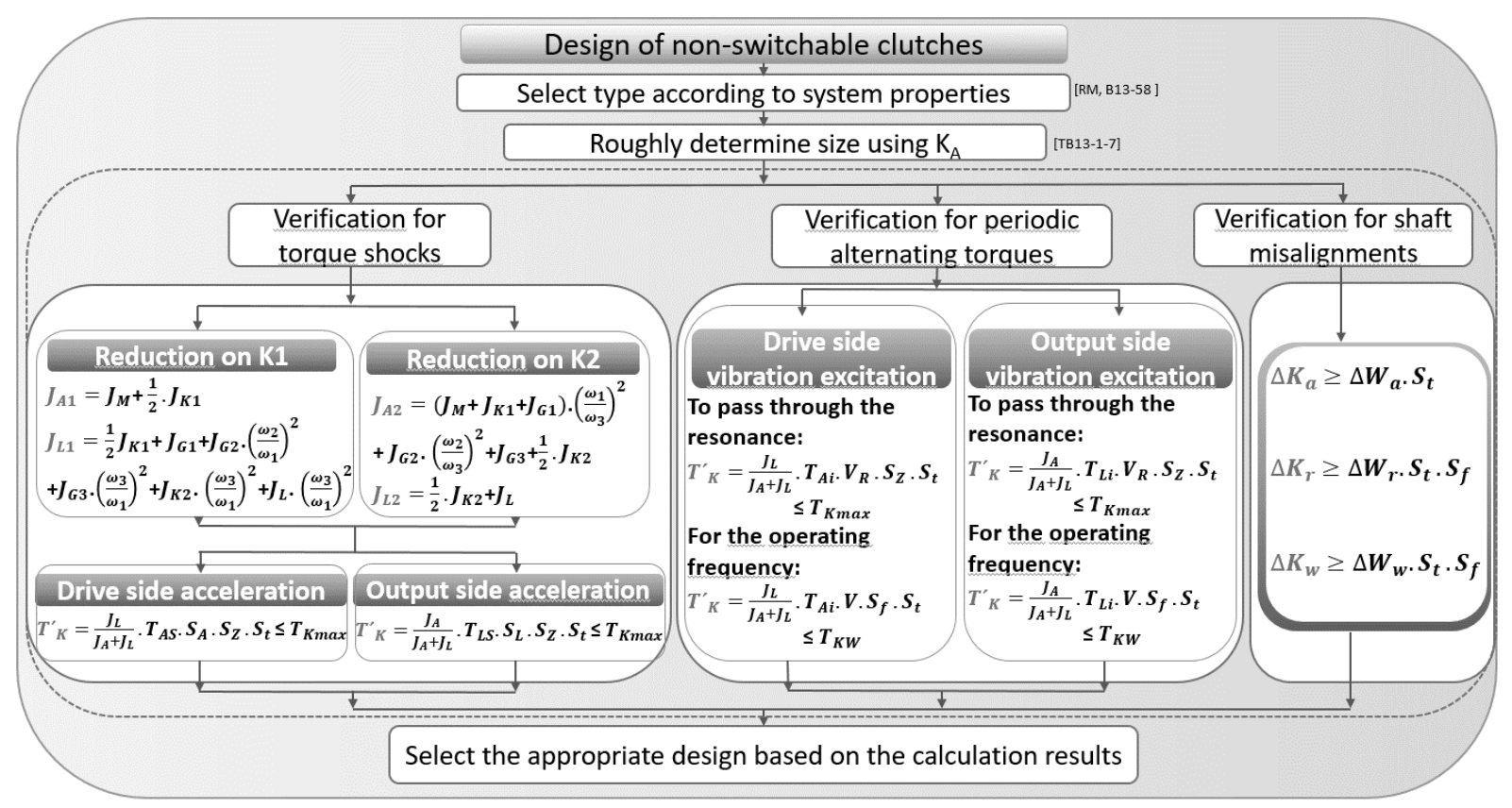

Figure 2: Design of non-switchable clutches 


\subsection{AR Exercise Task: Concept and Task}

As already specified in the objectives, the activating AR task will contain a combination of the four teaching methods, namely 2D, 3D, paper model and animation. After design of non-switchable clutches has been explained in the lecture, a handout with AR-ThingMark is deployed for the 2D part of the tasks. By presenting the task on the handout, students can keep all relevant information in view at all times, despite the low information density of the experience. Now the AR experiences are designed as multiplechoice exercises, which should cover all steps for the design of non-switchable clutches (see figure 2).

Figure 3 shows the concept of the first and second AR experience, which correspond to the first two steps in clutch design. The first one shows the different clutch types available for selection and the corresponding 3D models. After the design type has been determined, the 3D model of the observed system is displayed in the second experience. Similarly, in the third experience, the start-up of the system is shown as an animation and the multiple-choice task is displayed.
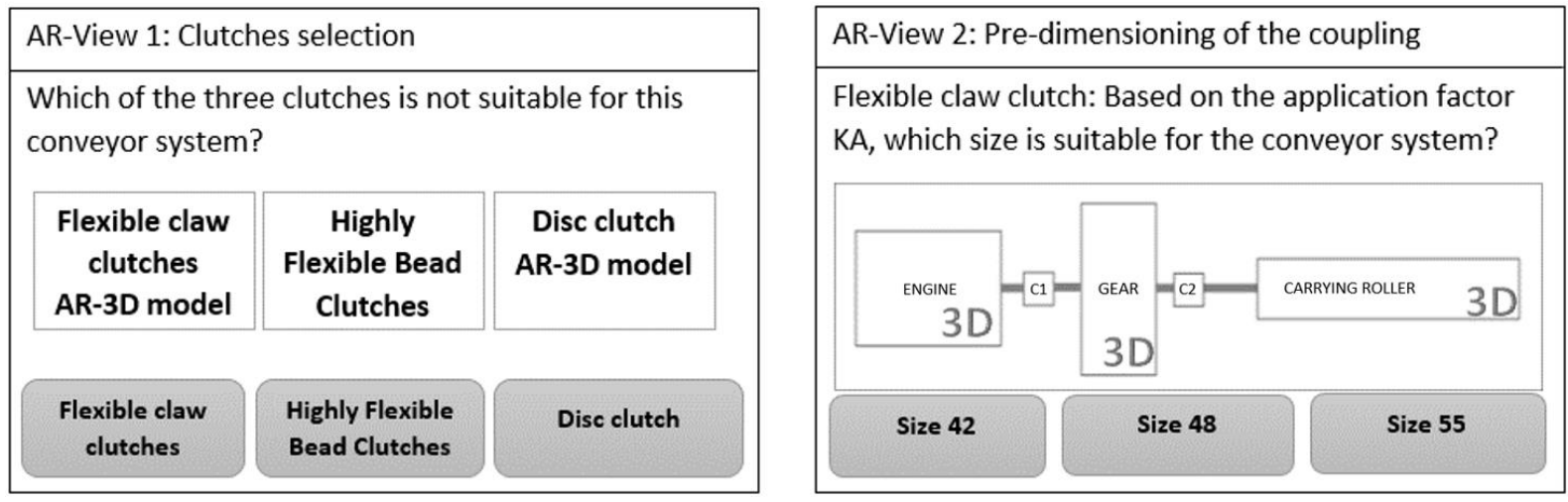

Figure 3: $A R$ experiences 1 and 2

In the next experience, a general animation on the subject of running through the critical speed is shown and the multiple-choice task is displayed. In the fifth experience, an animation of radial shaft displacement is shown and the multiple-choice task is used to check whether it is allowed. Next, the second experience is shown but the corresponding task has to be answered by the last three experiences. In the last experience, the 3D model of the conveyor system is displayed, but the elastic claw clutches are replaced by highly elastic bead clutches. Finally, students need to answer which clutch size is suitable for the second clutch in the system $\left(K_{2}\right)$. To answer this question, the students have to repeat all six previous experiences again and, analogously, perform the necessary calculations.

\subsection{Solution and distractors from the AR exercise}

The AR exercise task was solved according to Figure 2. The distractors that are integrated into the AR exercise task were derived from misconceptions or frequently observed mistakes by students, as specified in the objective. An example of misconceptions is, that all damping clutches has the magnification function $V_{R}$. This misconception results in an incorrect value of $T^{\prime}{ }_{K}$ for operating frequency and it thus taken as a distractor.

\subsection{Main steps creating the AR exercise}

As already defined in chapter 3, the AR exercise is created using the AR experience creation process. This process is based on the use of PTC software, namely Creo Parametric, Creo Illustrate and Vuforia Studio. The creation process serves as a guide for creating AR experiences, describing the main features and gadget to be used.

Following the creation process, the AR-creator uses Creo Parametric not only to design the CAD models, but also to animate movements of components such as the shafts of a gearbox. After the 3D model is created, it should be animated using the Mechanism function. In order to animate the system during work mode, the moving or rotating components should be integrated into the system as swivel joints during assembly. Unlike rigid components, components with the connection type "swivel joint" have a rotational degree of freedom. For rotating components, the animation can be set to, for example, speed, direction of rotation, gear ratio, frame rate, etc. Once the animation has been created, the assembly cannot be adjusted in Creo Parametric, otherwise the created animation will no longer be usable. 
By using Creo Illustrate, the existing animations were extended by the functions colorization, fade in and fade out, manipulation as well as editing and assembly work. Thus, these actions will be part of the animation, i.e. their development can be observed over time. The duration of the action can be set using a timeline. To draw the attention of the audience to a specific area of the animation, Creo Illustrate also provides the creator with a flashing function (see Figure 4). After the extended animation has been completed, it is now saved as a pvz file together with the corresponding 3D model.

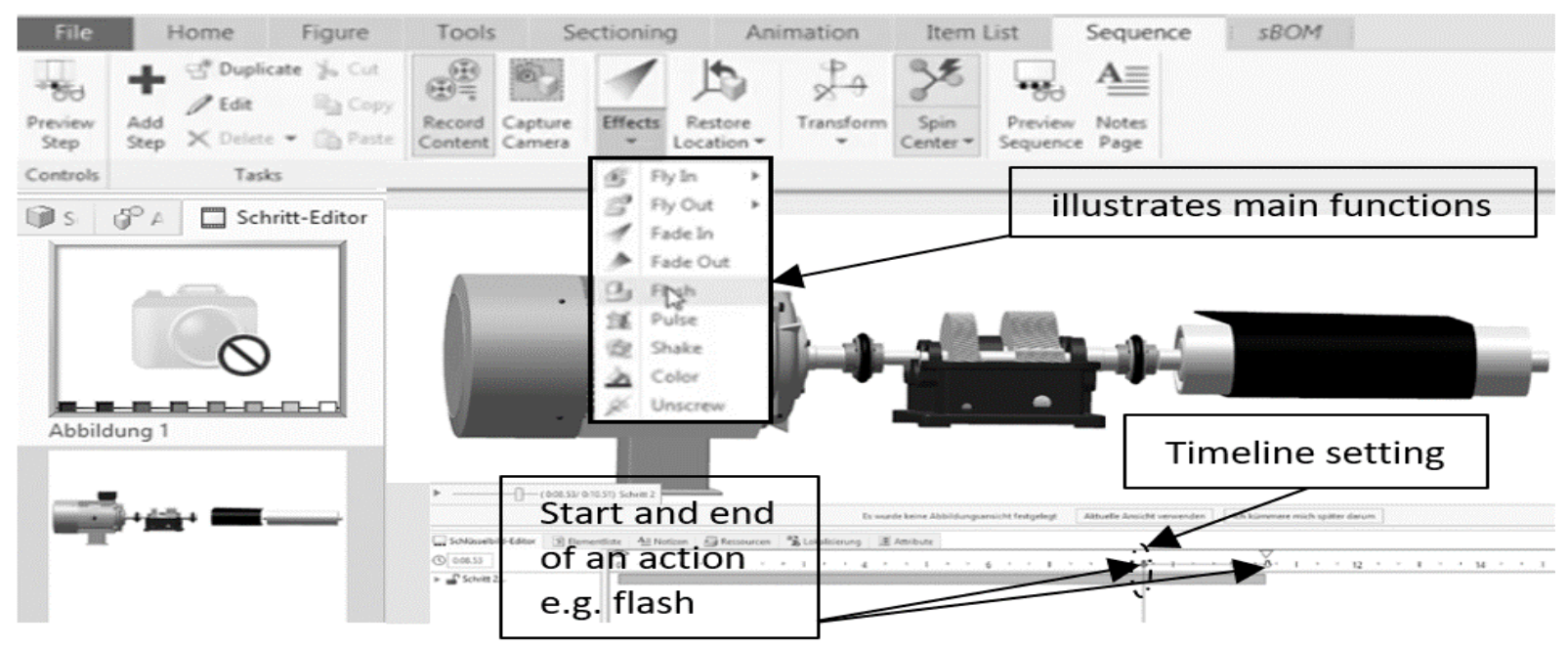

Figure 4: Main functions of Creo Illustrate

Then the creator uploads the created pvz files into the 3D environment of the Vuforia project. Next, virtual buttons are inserted in the $2 \mathrm{D}$ environment to control the fade-in and fade-out of animations, text fields, 3D bodies and even other control elements like sub-buttons. For example, Figure 5 shows one of seven views of the AR exercise (in the 3D environment). After pushing the corresponding selection button, the corresponding pvz file and multiple-choice task are displayed and activated.

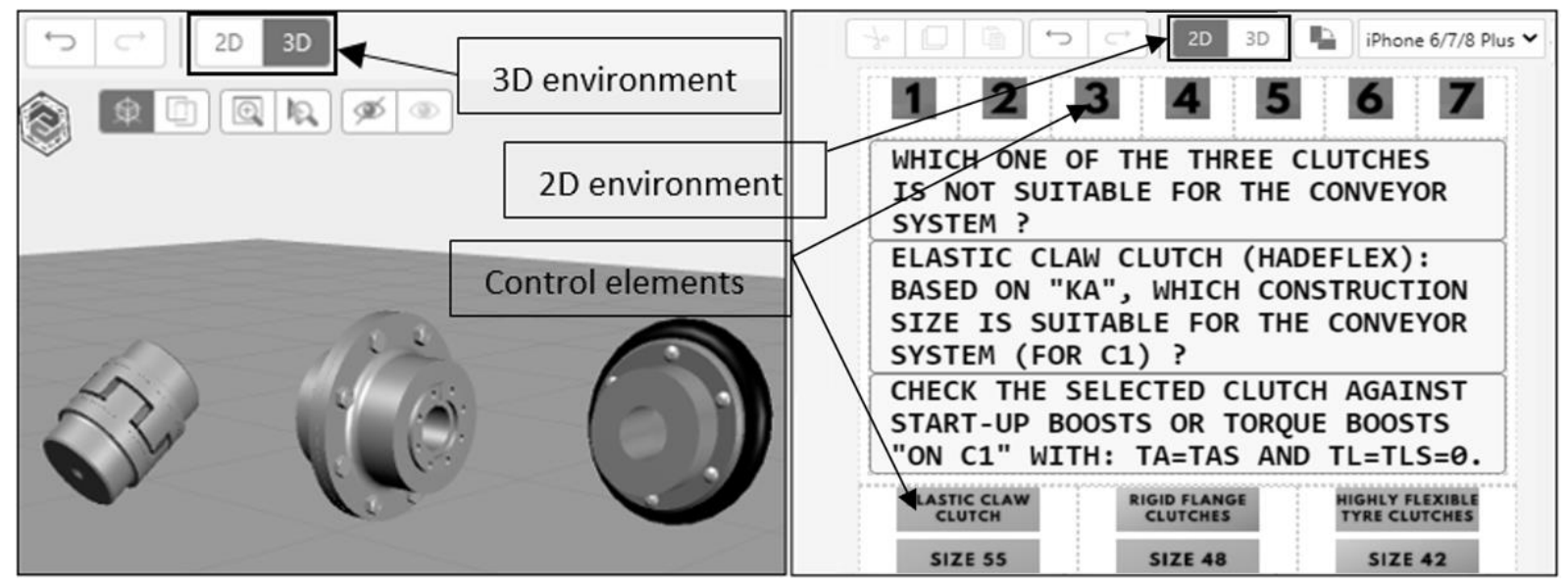

Figure 5: The 2D and 3D environment of Vuforia Studio

As no Multiple Choice Gadget has been provided in the Vuforia Studio Library, it must be constructed manually using toggle buttons and the drag and drop function. The switch buttons of the multiplechoice task are connected in series, so that after pressing a switch button (one of the answer options) the correct and incorrect answers can be indicated by changing colour and displaying feedback.

As shown in Figure 6, seven menu buttons are displayed above the animations or 3D objects ([1] [7]). They should show and activate each of the seven multiple-choice questions. For example, in the first AR view (the screenshot top left) the first task is active, as can be seen from the colour of the first menu button [1]. This means that the corresponding 3D objects and the three response options are also shown. After the user selects or clicks on an answer, the correct answer will be visually indicated (by changing the colour of the button). At the same time, a feedback is displayed at the bottom of the AR view to explain the solution or the errors. Afterwards the user should switch to the second task, i.e. click the button [2] and so on. 

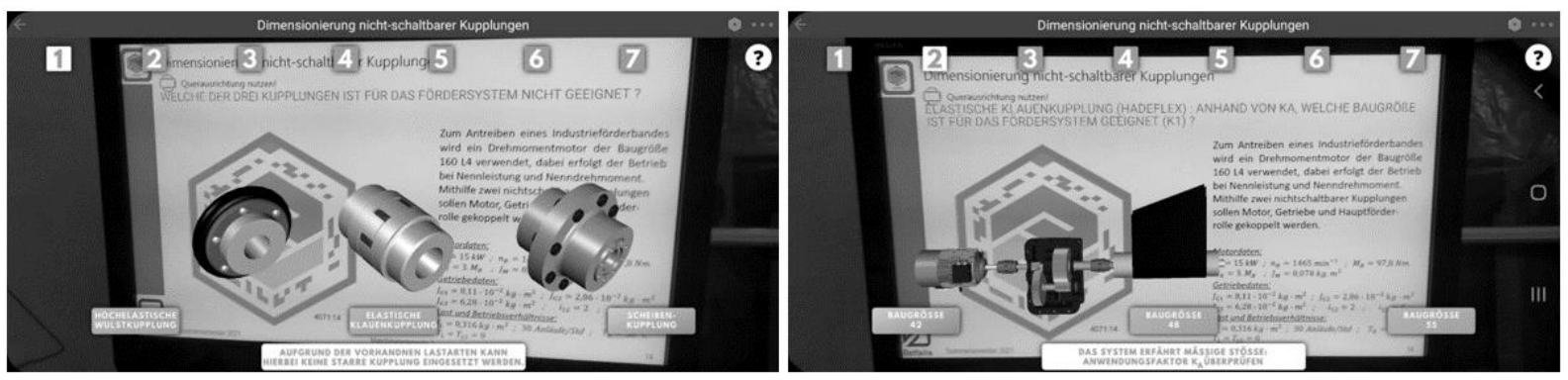

Figure 6: Screen shots from view 1 to 4

\section{EVALUATION BY THE STUDENTS}

For the evaluation of the created AR exercise, a survey has been carried out during the online lecture period. The survey needs to answer the following research questions:

- Has the understanding of the topic "design of non-switchable clutches" improved by working through the AR exercise?

- Did the AR exercise activate participation of students during the lecture?

- Did the AR exercise improve motivation in basic lectures?

Students were randomly organised in two groups. One group was enabled to use the AR-exercise. The other group had to learn with the normal learning material. The assessment of learning success was carried out by the exercise results for both groups. In the AR-group, all students (10 returns) had correct answers. In the control group (16 returns), just $81 \%$ of the students gave the correct answers. Due to the current situation, the sample is too small to derive general statements. However, it is an objective indicator that the learning success has been increased by the experience.

The students themselves assess the subjective influence. The students of the AR group rated the following statements on a Likert scale (see figure 7). In the Likert scale, "0" stands for not at all true and "10" for completely true. The evaluation results shown in the figure were formed from the average of the individual evaluations.

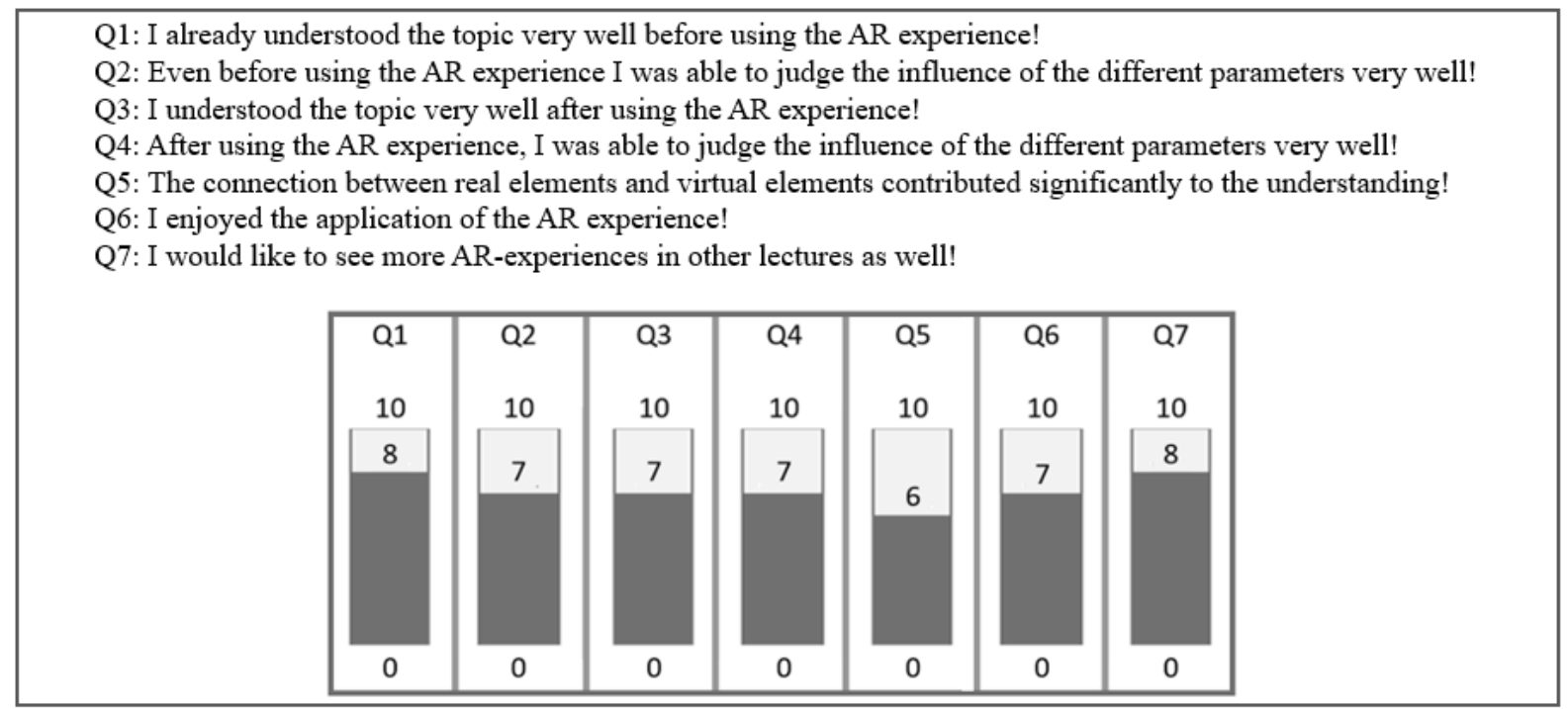

Figure 7: Evaluation by the students

The ratings of the first two questions show that most students already met the basic requirements for completing the task, namely prior knowledge, by giving scores close to 10 . The comparison of Q1 and Q2 with Q3 and Q4 shows that the students have the impression that the AR exercise has neither improved nor worsened comprehension. The authors assume that pandemic circumstances led to a high amount of students, who already started learning the topic in the last semester, but refused to write the exam. Due to the already high entry level, the increase in knowledge was rather low. Furthermore, the authors could not assess if students were more active. Further investigation is needed. 
The lowest rating appeared for the fifth question. In this exercise, real elements were used to reduce the information density of the experiences rather than to directly transmit understanding. The answers from the last two questions lead to the conclusion that students enjoyed the use of AR technology in teaching and would like to see more AR experiences in the future. Apart from learning outcomes, Q6 and Q7 show, that the AR-experience increased motivation. It is assumed that increased motivation led to better results.

\section{SUMMARY AND OUTLOOK}

As mentioned in the introduction, this paper was conducted as part of the Innovation-Plus project. The main goal of this project is to achieve efficient learning in higher education through the application of Augmented Reality. In order to achieve the main goal, it was concretized into several sub-goals. This paper focusses on the activation of students through the application of Augmented Reality technology. Due to the current situation caused by the corona pandemic, the topic covered has even become more attractive. This is because most of the courses are held online and therefore the students do not have enough opportunities to actively participate. Augmented Reality in general offers teaching support independent of time and place and this work in particular offers an opportunity for activation using ideas from JiTT and PI.

The created AR exercise deals with a complex topic from the machine element lecture. The created AR exercise consists of seven multiple-choice questions. The multiple-choice tasks require calculation work and the highest degree of Bloom's learning taxonomy. During the processing of these questions, simple 3D models as well as animations are displayed by using the AR technique.

The evaluation with a student group has shown that the goal "activation through AR" has been fulfilled and an important step towards efficient learning has been achieved. However, the sample is too small to derive general statements. More AR content will be created and transferred to different other lectures. With a wider application and larger samples, the efficiency of AR exercises can be assessed better and better in the future.

\section{ACKNOWLEDGEMENTS}

The authors gratefully thank the Ministry of Science and Culture of the German state of Lower Saxony for funding the project "Innovation Plus - Augmented Reality Experiences for Higher Education".

\section{REFERENCES}

Azuma, Ronald T. (1997): A Survey of Augmented Reality. In: Presence: Teleoperators \& Virtual Environments 6 (4), S. 355-385. https://dx.doi.org/10.1162/pres.1997.6.4.355.

Balzerkiewitz, H.-P.; Stechert, C. (2020): The evolution of virtual reality towards the usage in early design phases. In: Proc. Des. Soc.: Des. Conf. 1, S. 91-100. https://dx.doi.org/10.1017/dsd.2020.159.

Bauer, Thomas (2019): Peer Instruction als Instrument zur Aktivierung von Studierenden in mathematischen Übungsgruppen. In: Math Semesterber 66 (2), S. 219-241. https://dx.doi.org/10.1007/s00591-018-0225-8.

Bloom, Benjamin Samuel (1956): Taxonomy of educational objectives. New York, London: McKay; Longman.

Chlebusch, J.; Köhler, I.; Stechert, C. (2020): Reasonable application of augmented reality in engineering education. In: Proc. Des. Soc.: Des. Conf. 1, S. 1677-1686. https://dx.doi.org/10.1017/dsd.2020.62.

Fotis Liarokapis et al. (2004): Web3D and augmented reality to support engineering education. Article in World Transactions on Engineering and Technology Education · January 2004, S. 10-14. Online verfügbar unter https://www.researchgate.net/publication/38174320.

Lee, Kangdon (2012): Augmented Reality in Education and Training. In: Techtrends tech trends 56 (2), S. 1321. https://dx.doi.org/10.1007/s11528-012-0559-3.

Marrs, Kathleen A.; Novak, Gregor (Hg.) (2004): Just-in-Time Teaching in biology: creating an active learner classroom using the Internet (3).

Mazur, Eric (1997): Peer Instruction: A User's Manual. Upper Saddle River, NJ: Prentice Hall (67).

Novak, Gregor M. (1999): Just-in-time teaching. Blending active learning with web technology. Upper Saddle River, NJ: Prentice Hall (Prentice Hall series in educational innovation).

Waldherr, Franz; Walter, Claudia (2014): Didaktisch und praktisch. Ideen und Methoden für die Hochschullehre. Zweiten Auflage. Stuttgart [Germany]: Schäffer-Poeschel Verlag.

Wolf, Kathrin; Nissler, Antje; Eich-Soellner, Edda; Fischer, Rainer (2014): Mitmachen erwünscht - aktivierende Lehre mit Peer Instruction und Just-in-Time Teaching. In: ZFHE 9 (4). https://dx.doi.org/10.3217/zfhe-904/09. 